\title{
Differential Distributed Space-Time Modulation for Cooperative Networks
}

\author{
Genyuan Wang, Member, IEEE, Yimin Zhang, Senior Member, IEEE, and Moeness Amin, Fellow, IEEE
}

\begin{abstract}
In this paper, we develop a protocol for the construction of cooperative networks when the channel state information is not available at the transmitters and the receivers. In the proposed protocol, differential space-time codewords are generated at the source terminal. In the broadcast phase, each row of the differential space-time codeword is transmitted to a different relay, whereas in the relay phase, the relaying terminals retransmit the codeword through simple amplify-andforward algorithm. The performance of the cooperative diversity system is analyzed for a two-user case for different channel environments in terms of the diversity gain and the diversity product. The optimization of the power allocation between source and relay terminals is considered for the maximization of the diversity product. When the same modulation scheme is used, the performance of differential detection is degraded by $3 \mathrm{~dB}$ noise enhancement compared with coherent detection.
\end{abstract}

Index Terms-Cooperative diversity, MIMO, space-time coding, differential coding.

\section{INTRODUCTION}

$\mathbf{T}$ HE multi-input-multi-output (MIMO) technology, incorporating appropriate space-time coding schemes, allows considerable increase of the information capacity in fading channels [1]-[4]. Without increasing premium bandwidth or transmit power, a MIMO system can achieve higher diversity gains and data rates, compared to single-input or/and singleoutput system counterparts.

It is often impractical, however, for a transmitter to host a large number of antennas for a desirable diversity gain, especially for small size transceivers operating at relatively low frequencies. In addition, delivery of information from a transmitter to a receiver can be compromised when they are distant apart, or when the wireless channel links are highly impairing. This difficulty arises in a wide class of wireless networks, such as local area networks (LANs), sensor networks, and ad hoc networks. For these applications, cooperative diversity exploiting cooperation among multiple terminals proves desirable.

The cooperative diversity techniques have recently attracted considerable attentions. In responding to the increasing needs of effective and reliable wireless networks in various applications, the development of cooperative diversity techniques

Manuscript received October 20, 2004; revised June 14, 2005 and March 7, 2006; accepted July 20, 2006. The associate editor coordinating the review of this paper and approving it for publication was S. Aissa. This work was supported in part by the ONR under Grant No. N00014-04-1-0617, ONR/NSWC under contract No. N65540-05-C-0028, and NSF under grant No. EEC-0332490.

The authors are with the Center for Advanced Communications, Villanova University, Villanova, PA 19085, USA (e-mail: \{genyuan.wang, yimin.zhang, moeness.amin\}@villanova.edu).

Digital Object Identifier 10.1109/TWC.2006.04714. has benefitted from the recent advances of space-time codes, transmit diversity, and MIMO technologies. Recent research work has shown system feasibility and provided capacity analyses of different cooperative diversity techniques (see for example, [5]-[21]). These methods assume that channel state information (CSI) is available at the receivers, although few assume CSI knowledge at the transmitters.

CSI knowledge at the receivers is usually obtained through channel estimation, either using training (pilot) signals or utilizing blind methods. However, channel estimation is often complicated and may reduce the transmission efficiency, if pilot signals are used. In addition, the estimation becomes unreliable and impractical in fast fading environments [22]. In such situations, MIMO systems often adopt differential spacetime coding schemes which use differential codes between two adjacent space-time code matrices so that the decoding at the receiver is independent of the fading channels [22]-[30].

As the cooperative diversity schemes involve both broadcast and relay phases, multiple cooperative terminals should be considered. The propagation channels are, therefore, more complicated than those encountered in MIMO scenarios [31]. Accordingly, the consideration of system design without assuming knowledge of CSI at a receiver becomes a practical requirement.

So far, several differential cooperative diversity schemes have been developed for applications in the absence of CSI knowledge at the receivers. A repetition-based differential amplify-and-forward scheme is proposed in [32] for a singlerelay scenario, where differentially encoded BPSK signals are used. In [33], a differential modulation scheme is proposed for two-user cooperative diversity systems, where the relay terminals use QPSK modulation to transmit two BPSK streams. The information of one user stays in the in-phase axis (Iaxis) while that of the other user stays in the quadrature-phase axis (Q-axis). In [34], a non-coherent decode-and-forward scheme is developed for binary frequency shift keying (BFSK) modulations.

In this paper, we develop a new distributed space-time modulation scheme for cooperative systems that require no knowledge of the CSI at both transmitters and receivers. In the proposed protocol, differential space-time codewords are generated at the source terminal, and each row of a codeword is relayed by a different relay terminal. Fundamentally, any differential space-time codes can be employed in the proposed scheme, whereas the relay terminals implement a simple amplify-and-forward relaying algorithm. An entry of the user's differential space-time codeword is not based on any specific modulation such as PSK or FSK. The performance of the 
cooperative diversity system is analyzed for a two-user case for different channel environments in terms of the diversity gain and the diversity product. The optimization of the power allocation between source and relay terminals are considered for the maximization of the diversity product. Roughly, the performance of differential detection is degraded by $3 \mathrm{~dB}$ noise enhancement compared with the coherent detection, when the same modulation scheme is used.

In this paper, we consider memoryless channels and assume that the terminals transmitting the same distributed space-time codeword are synchronized at the destination receiver. When the channels are not perfectly synchronized, the synchronization error between the terminals can be considered as channel dispersion effect [35]. The effect of channel dispersion in cooperative network as well as the effective approaches using equalization and orthogonal frequency division multiplexing (OFDM) techniques have been considered in [35]-[37].

This paper is organized as follows. In Section II, the system model is introduced. In Section III, we review the existing cooperative protocols, and then propose a new protocol which is effective to transmit distributed information when the CSI is unavailable at the receivers. The differential space-time cooperative structure is shown in Section IV and the performance analysis is provided in Section V. Numerical results are provided in Section VI.

\section{SySTEM MODEL}

To illustrate the concept of cooperative diversity in a wireless network, consider a simple narrowband communication model as depicted in Fig. 1(a). Each user cooperates with the other users and serves as a relay terminal for them. Therefore, each user receives an attenuated and noisy version of the partners' transmitted signal and relays it to the destination or other relays. The destination terminal receives a noisy version of the sum of the attenuated signals from all users (see Fig. 1(b)). Denote the number of users as $M$. The received signal at the destination during the $l$ th symbol period is expressed in the baseband model as

$$
y(l)=\sum_{i=1}^{M} \gamma_{i 0} h_{i 0}(l) x_{i}(l)+n(l),
$$

where we used subscript 0 to denote the destination terminal for notational convenience. In $(1), x_{i}(l)$ is the source or relaying signal transmitted by the $i$ th user, for $i=1,2, \cdots, M$, $\gamma_{i 0}$ is the large-scale attenuation factor of the channel between the $i$ th terminal and the destination, whereas $h_{i 0}(l)$ represents the respective unit-variance small-scale time-varying statistics of the channel. In addition, $n_{0}(l)$ is the additive channel noise term at the destination.

It is assumed that a relay terminal does not receive signals from other users when it is transmitting. Therefore, the signal received at relay terminal $k$ is expressed as

$$
r^{(k)}(l)=\sum_{i \neq k} \gamma_{i k} h_{i k}(l) x_{i}(l)+n_{r}^{(k)}(l) .
$$

where $\gamma_{i k}$ and $h_{i k}(l)$, respectively, represent the large-scale attenuation factor and the small-scale time-varying statistics of the channel between user terminals $i$ and $k$, and $n_{r}^{(k)}(l)$ is

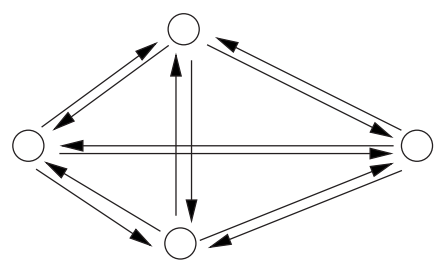

(a)

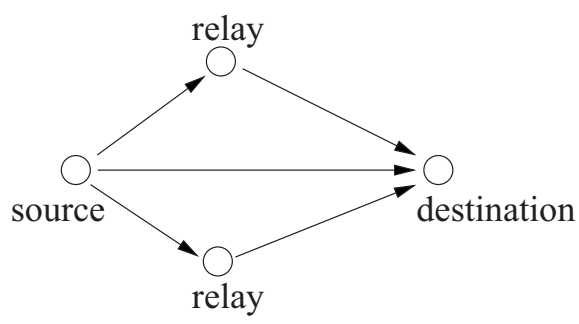

(b)

Fig. 1. System model.

the additive channel noise term at the relay receiver $k$, where $i, k=1,2, \cdots, M$.

Depending on how the relay terminals relay the signals from other users, two major schemes can be devised, namely, amplify-and-forward and decode-and-forward. In this paper, we focus on the simpler amplify-and-forward scheme which requires the least complexity at the relay terminals.

\section{Cooperation Protocols}

\section{A. Existing Protocols}

Several cooperative diversity protocols have been investigated. The early protocols used repetition-based approaches, which is illustrated in Fig. 2(a) [8], [19]. After source terminal $i$ broadcasts a block of information symbols (e.g., $\mathbf{s}_{i}(l)=$ $\left[s_{i}(l), s_{i}(l+1), \cdots, s_{i}(l+L-1)\right]$, where $L$ is the length of the data block) to the destination and the relay terminals, all relay terminals repeat the same data (i.e., $\mathbf{s}_{i}(l)=\left[s_{i}(l), s_{i}(l+\right.$ $\left.\left.1), \cdots, s_{i}(l+L-1)\right]\right)$ in a sequential order.

Recently, more effective protocols have been developed to take advantages of the advances of MIMO space-time codes. For example, [8] and [19] proposed space-time cooperation protocols which are illustrated in Fig. 2(b) and (c). In the broadcast phase, the source terminal $i$ broadcasts a block of information symbols (e.g., $\mathbf{s}_{i}(l)=\left[s_{i}(l), s_{i}(l+1), \cdots, s_{i}(l+\right.$ $L-1)]$ ) to the destination and the relay terminals. In the relay phase, for scheme I depicted in Fig. 2(b), the source and the relay terminals transmit different rows of a space-time codeword $\mathbf{X}_{i}(\nu)$, which is constructed from $\mathbf{s}_{i}(l)$, where $\nu$ denote the index of a coded data block. Space-time cooperative scheme II is very similar to scheme I. The only difference between them lies in the fact that in scheme I, the source terminal takes part in the relay phase, whereas in scheme II it does not. Such protocols provide more effective bandwidth use, particularly when the number of cooperative users is large.

\section{B. Proposed Protocol}

Most existing cooperative protocols assume that the CSI is known at the receivers. In this section, we develop a 


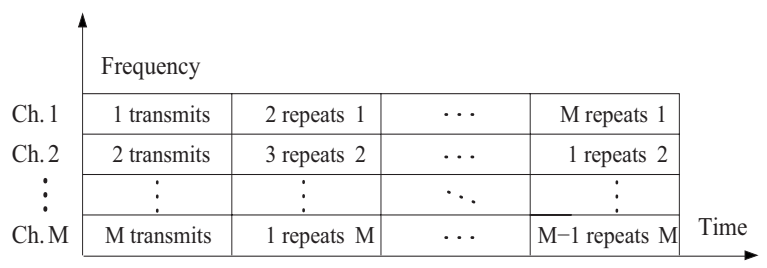

(a) repetition-based diversity scheme

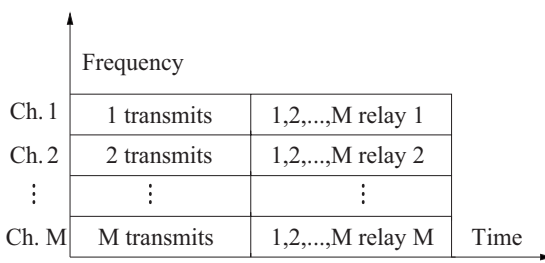

(b) space-time cooperation scheme I

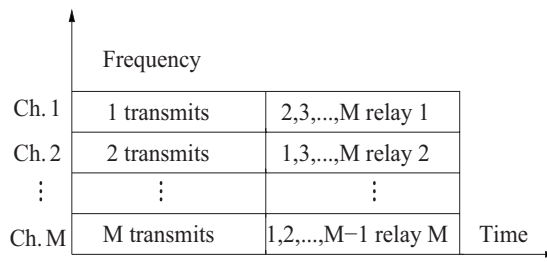

(c) space-time cooperation scheme II

Fig. 2. Cooperative diversity schemes.

\begin{tabular}{c|c|c|} 
& \multicolumn{2}{|c}{ Frequency } \\
Ch. 1 & 1 transmits & $2,3, \ldots, \mathrm{M}$ repeat 1 \\
\cline { 2 - 3 } Ch. 2 & 2 transmits & $1,3, \ldots, \mathrm{M}$ repeat 2 \\
\cline { 2 - 3 } & $\vdots$ & $\vdots$ \\
\cline { 2 - 3 } Ch. M & M transmits & $1,2, \ldots, \mathrm{M}-1$ repeat $\mathrm{M}$ \\
\cline { 3 - 3 } Time \\
\hline
\end{tabular}

Fig. 3. The proposed space-time cooperation scheme.

protocol that is applicable to space-time cooperation, where the CSI is unavailable at the receivers. The proposed protocol performs differential distributed space-time coding and information detection using the relatively simple amplify-andforward algorithm. Fig. 3 depicts the proposed protocol. When considering user $i$ as the source user, the other $M-1$ ones act as its relays. At the source terminal, an $M \times M$ spacetime codeword, $\mathbf{X}_{i}(\nu)$, is formed from the source information symbols. Each of the $M-1$ relay terminals receives a different row of the codeword $\mathbf{X}_{i}(\nu)$ that is transmitted from the source user through the broadcast phase (i.e., during the first $M-1$ time blocks). All the $M$ rows of $\mathbf{X}_{i}(\nu)$ are transmitted from the $M$ terminals in the relay phase which spans the $M$ th time block. It is noted that the destination listens to the source during the broadcast phase, whereas it listens to the source and the relay terminals during the relay phase.

Consider the simple case of two users, i.e., $M=2$. The source terminal forms a $2 \times 2$ MIMO space-time codeword $\mathbf{X}_{i}(\nu)$ from the source information symbols $\mathbf{s}_{i}(l)=$ $\left[s_{i}(l), s_{i}(l+1), \cdots, s_{i}(l+L-1)\right]$. Then, using the cooperative protocol, the source and relay terminals equivalently transmit the following $2 \times 4$ codeword $\hat{\mathbf{X}}_{i}^{\prime}(\nu)$ in the cooperative diversity system, i.e.,

$$
\begin{aligned}
\mathbf{X}_{i}(\nu) & =\left[\begin{array}{ll}
x_{i}(\nu, 1) & x_{i}(\nu, 2) \\
x_{i}(\nu, 3) & x_{i}(\nu, 4)
\end{array}\right] \\
\Rightarrow & \hat{\mathbf{X}}_{i}^{\prime}(\nu)=\left[\begin{array}{cccc}
x_{i}(\nu, 3) & x_{i}(\nu, 4) & x_{i}(\nu, 1) & x_{i}(\nu, 2) \\
0 & 0 & \hat{x}_{i}(\nu, 3) & \hat{x}_{i}(\nu, 4)
\end{array}\right],
\end{aligned}
$$

where $\hat{x}_{i}(\nu, \tau)$ is the complex scaled and noisy version of $x_{i}(\nu, \tau)$. In the above space-time codewords, the columns specify the time dimension, whereas the rows specify the space dimension (i.e., antennas or relay terminals).

\section{Differential Cooperation Schemes}

In this section, we first briefly review the differential spacetime codes developed for MIMO systems, and then show the feasibility of employing them in the space-time cooperation applications through the proposed protocol.

\section{A. Differential Space-Time Codes}

In order to deal with rapid MIMO fading environments where the CSI is unavailable at the receiver, differential spacetime codes can be used. For essence, differential coding between two adjacent space-time code matrices is used so that the decoding at the receiver can be performed without the knowledge of channels [22]-[30]. Differential space-time codes are most commonly used in the form of unitary matrices [22], [27].

Let $\mathcal{G}=\left\{\mathbf{G}_{i}, i=1, \cdots, N\right\}$ be a set of $M \times M$ unitary matrices (i.e., $\mathbf{G}_{i} \mathbf{G}_{i}^{H}=\mathbf{G}_{i}^{H} \mathbf{G}_{i}=\mathbf{I}_{M}$, where $\mathbf{I}_{M}$ is the $M \times$ $M$ identity matrix). $\log _{2} N$ information bits are mapped to one of the $N$ unitary matrices $\mathbf{G}_{i}$ in $\mathcal{G}$. To send the message $\mathbf{G}(\nu) \in \mathcal{G}$ over time block $\nu$, the transmitter sends an $M \times M$ matrix $\mathbf{C}(\nu)$ where

$$
\mathbf{C}(\nu)=\mathbf{C}(\nu-1) \mathbf{G}(\nu)=\mathbf{C}(0) \prod_{\tau=1}^{\nu} \mathbf{G}(\tau),
$$

and $\mathbf{C}(0)$ is the initial value of $\mathbf{C}(\nu)$. Matrix $\mathbf{C}(\nu)$ takes the following general format

$$
\mathbf{C}(\nu)=\left[\begin{array}{cccc}
c_{11}(\nu) & c_{12}(\nu) & \ldots & c_{1 M}(\nu) \\
c_{21}(\nu) & c_{22}(\nu) & \ldots & c_{2 M}(\nu) \\
\vdots & \vdots & \ddots & \vdots \\
c_{M 1}(\nu) & c_{M 2}(\nu) & \ldots & c_{M M}(\nu)
\end{array}\right]
$$

The spectral efficiency of this code is $(1 / M) \log _{2} N$ bits per channel use. When the channels corresponding to different transmit antennas are independent and identically distributed (i.i.d.), $\mathbf{C}(\nu)$ is often designed to take the form of unitary matrices for improved system capacity [23], [24], [26]. That is,

$$
\mathbf{C}(\nu) \mathbf{C}^{H}(\nu)=\mathbf{I}_{M} .
$$

In this case, $\mathbf{C}(0)$ is chosen as unitary.

The differential space-time coding scheme works well in time-varying channels, provided that the channels do not change significantly over a period of two adjacent codewords, i.e., $\mathbf{h}(\nu-1)=\mathbf{h}(\nu)$, where $\mathbf{h}(\nu)=\left[h_{10}(\nu), \cdots, h_{M 0}(\nu)\right]$ is a vector channel with the $i$ th element representing the 
channel coefficient between the $i$ th transmit antenna and the destination receiver. Denote the signals received at the destination receiver corresponding to the $(\nu-1)$ th and $\nu$ th codewords as

$$
\mathbf{y}(\nu-1)=\mathbf{h}(\nu-1) \mathbf{C}(\nu-1)+\mathbf{n}(\nu-1)
$$

and

$$
\mathbf{y}(\nu)=\mathbf{h}(\nu) \mathbf{C}(\nu)+\mathbf{n}(\nu)
$$

then we have

$$
\begin{aligned}
\mathbf{y}(\nu) & =\mathbf{h}(\nu-1) \mathbf{C}(\nu-1) \mathbf{G}(\nu)+\mathbf{n}(\nu) \\
& =[\mathbf{y}(\nu-1)-\mathbf{n}(\nu-1)] \mathbf{G}(\nu)+\mathbf{n}(\nu) .
\end{aligned}
$$

Because $\mathbf{G}(\nu)$ is unitary, $-\mathbf{n}(\nu-1) \mathbf{G}(\nu)$ can be considered as additional noise term with the same variance as $\mathbf{n}(\nu)$. Therefore, the information $\mathbf{G}(\nu)$ can be recovered from $\mathbf{y}(\nu-$ $1)$ and $\mathbf{y}(\nu)$ through the above relationship at the expense that the equivalent noise power is doubled.

\section{B. Differential Space-Time Cooperative Schemes}

We now consider the feasibility of extending the differential space-time codes to cooperative networks. For the clarity of the presentation of the proposed method, we ignore the noise effect in this section. The effect of noise, and the optimization of power allocation in different antennas as well as different broadcast and relay phases, will be considered in Section V for a two-user scenario.

Assume that there are $M$ users cooperating with each other. Without loss of generality, we only focus on the first user's information and the user index is omitted without confusion. Following the protocol depicted in Section III, $\mathbf{C}^{\prime} \in \mathcal{C}^{M \times M^{2}}$ is constructed from the corresponding differential space-time codeword $\mathbf{C} \in \mathcal{G}$. Because different rows of the distributed space-time code (DSTC) codeword undergo different propagation channels with different attenuations, it is desirable to allocate energy adaptively to different rows. For this purpose, the DSTC is expressed in the generalized format as expressed in equation (8), shown at the top of the next page ${ }^{1}$, where $P_{i} \geq 0$ is the transmit power of different users, $i=1, \cdots, M$, and $\alpha_{i} \geq 0$ is used to adjust the transmit power at the source terminal corresponding to different codeword rows. Because $\mathbf{C}(\nu)$ is unitary, $\sum_{k=1}^{M}\left|c_{i k}\right|^{2}=1$ for $i=1, \cdots, M$. Therefore, the total codeword power of the DSTC in (8) is $P=P_{1} \sum_{i=1}^{M} \alpha_{i}^{2}+\sum_{i=2}^{M} P_{i}$. The optimization of the power allocation is considered in Section V. It is assumed that signals transmitted from different terminals are synchronized at the destination receiver.
Next, we consider the detection of differential DSTC codeword $\mathbf{C}^{\prime}(\nu)$ without using the CSI. From (4) and (8), the relationship between two adjacent differential DSTC codewords, $\mathbf{C}^{\prime}(\nu)$ and $\mathbf{C}^{\prime}(\nu-1)$, and the information codeword $\mathbf{G}(\nu)$ is expressed as

$$
\mathbf{C}^{\prime}(\nu)=\mathbf{C}^{\prime}(\nu-1) \mathbf{G}^{\prime}(\nu)=\mathbf{C}^{\prime}(0) \prod_{\tau=1}^{\nu} \mathbf{G}^{\prime}(\tau),
$$

where

$$
\mathbf{G}^{\prime}(\nu)=\operatorname{diag}[\mathbf{G}(\nu), \cdots, \mathbf{G}(\nu)]=\mathbf{I}_{M} \otimes \mathbf{G}(\nu),
$$

with $\otimes$ denoting the Kronecker product operator. Because $\mathbf{G}(\nu)$ is unitary, it is clear that the $M^{2} \times M^{2}$ matrix $\mathbf{G}^{\prime}(\nu)$ is also unitary (i.e., $\mathbf{G}^{\prime}(\nu)\left(\mathbf{G}^{\prime}(\nu)\right)^{H}=\mathbf{I}_{M^{2}}$ ).

Note that the encoding process of (9) is performed at the source terminal and, therefore, it does not require the relay terminals to perform encoding or to possess information required in the differential coding process. In addition, it is noted that the distributed space-time codeword $\mathbf{C}^{\prime}(\nu)$ is, in general, not unitary. However, because $\mathbf{G}^{\prime}(\nu)$ is unitary, we have

$$
\begin{aligned}
\mathbf{C}^{\prime}(\nu)\left[\mathbf{C}^{\prime}(\nu)\right]^{H} & =\mathbf{C}^{\prime}(\nu-1) \mathbf{G}^{\prime}(\nu)\left[\mathbf{G}^{\prime}(\nu)\right]^{H}\left[\mathbf{C}^{\prime}(\nu-1)\right]^{H} \\
& =\mathbf{C}^{\prime}(\nu-1)\left[\mathbf{C}^{\prime}(\nu-1)\right]^{H} \\
& =\mathbf{C}^{\prime}(0)\left[\mathbf{C}^{\prime}(0)\right]^{H}
\end{aligned}
$$

Therefore, the transmit power of $\mathbf{C}^{\prime}(\nu)$ is unchanged for different $\nu$ and, subsequently, the robustness of the differential code is guaranteed.

Denote $\tilde{\mathbf{h}}(\nu)=\left[h_{10}(\nu), h_{12}(\nu) h_{20}(\nu), \cdots, h_{1, M}(\nu) h_{M, 0}(\nu)\right]$ as the $1 \times M$ channel vector whose components are assumed constant for any two adjacent codewords (i.e., $\tilde{\mathbf{h}}(\nu)=\tilde{\mathbf{h}}(\nu-1))$ and are statistically independent over time. Then, the $1 \times M^{2}$ received signal vector at the destination terminal is given by

$$
\begin{aligned}
\mathbf{y}(\nu) & =\tilde{\mathbf{h}}(\nu) \mathbf{C}^{\prime}(\nu)=\tilde{\mathbf{h}}(\nu) \mathbf{C}^{\prime}(\nu-1) \mathbf{G}^{\prime}(\nu) \\
& =\tilde{\mathbf{h}}(\nu-1) \mathbf{C}^{\prime}(\nu-1) \mathbf{G}^{\prime}(\nu)=\mathbf{y}(\nu-1) \mathbf{G}^{\prime}(\nu) .
\end{aligned}
$$

From (12), it becomes evident that the differential detection is feasible in cooperative networks because $\mathbf{G}^{\prime}(\nu)$ and, equivalently, $\mathbf{G}(\nu)$, can be recovered from $\mathbf{y}(\nu)$ and $\mathbf{y}(\nu-1)$, without the knowledge of the channel characteristics.

\footnotetext{
${ }^{1}$ An alternative and more general format of the codeword can take the following format (factors for power allocation are omitted for simplicity):

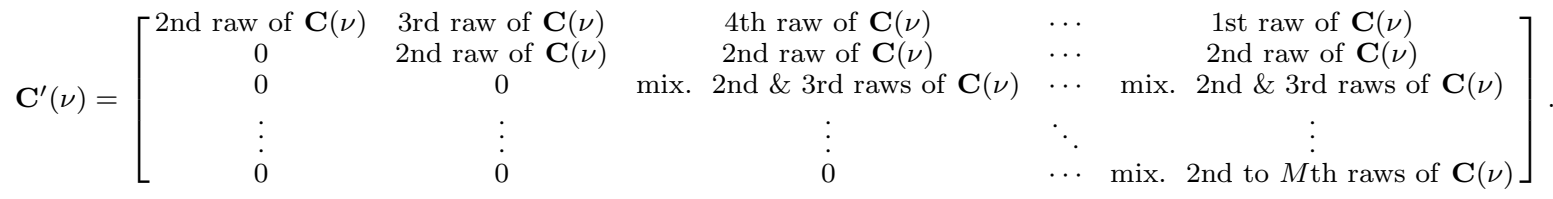




$$
\mathbf{C}^{\prime}(\nu)=\left[\begin{array}{cccccc}
\alpha_{2} \sqrt{P_{1}} c_{21}(\nu) & \cdots & \alpha_{M} \sqrt{P_{1}} c_{M M}(\nu) & \alpha_{1} \sqrt{P_{1}} c_{11}(\nu) & \cdots & \alpha_{1} \sqrt{P_{1}} \hat{c}_{1 M}(\nu) \\
0 & \cdots & 0 & \sqrt{P_{2}} c_{21}(\nu) & \cdots & \sqrt{P_{2}} c_{2 M}(\nu) \\
\vdots & \vdots & \vdots & \vdots & \ddots & \vdots \\
0 & \cdots & 0 & \sqrt{P_{M}} c_{M 1}(\nu) & \cdots & \sqrt{P_{M}} c_{M M}(\nu)
\end{array}\right]
$$

\section{Performance Analysis}

\section{A. Signal Model}

In this section, we consider the performance of the proposed system. For simplicity, we only consider a two-user scenario (ref. Fig. 4), while the extension to multi-user scenarios is straightforward. Because of the symmetry, we focus only on the transmission of user 1's information, whereas the second user is regarded as the relay.

Consider the generalized codeword $\hat{\mathbf{C}}^{\prime}(\nu)$ which considers the relay noise and takes the following form

$$
\begin{aligned}
& \hat{\mathbf{C}}^{\prime}(\nu) \\
& =\left[\begin{array}{cccc}
\sqrt{P_{1}} c_{21}(\nu) & \sqrt{P_{1}} c_{22}(\nu) & \alpha \sqrt{P_{1}} c_{11}(\nu) & \alpha \sqrt{P_{1}} c_{12}(\nu) \\
0 & 0 & \sqrt{P_{2}} c_{21}(\nu) & \sqrt{P_{2}} \hat{c}_{22}(\nu)
\end{array}\right],
\end{aligned}
$$

where $\hat{c}_{i k}$ denotes the attenuated and noisy version of $c_{i k}$, $i, k=1,2$. The expression of $\hat{c}_{i k}$ will be implicitly given later in (16). The total energy used to transmit $\hat{\mathbf{C}}^{\prime}(\nu)$ is $P=$ $\left(1+\alpha^{2}\right) P_{1}+P_{2}$. Note that, while we only use a single $\alpha$ $(\alpha \geq 0)$ in the first row (compared to $\alpha_{1}$ and $\alpha_{2}$ in (8)), it is obvious that $\alpha, P_{1}$, and $P_{2}$ together provide enough degreesof-freedom to adjust the power over different terminals and different time slots.

We first consider the signal flow during time block $\nu$ which spans four time slots. In the first two time slots, $c_{21}(\nu)$ and $c_{22}(\nu)$ are transmitted from user 1's antenna. As the result, the signals received at relay terminal (user 2) and the destination receiver are expressed, respectively, as

$$
\begin{aligned}
& {\left[r_{1}(\nu), r_{2}(\nu)\right]} \\
& =\sqrt{P_{1}} \gamma_{12} h_{12}(\nu)\left[c_{21}(\nu), c_{22}(\nu)\right]+\left[n_{r 1}(\nu), n_{r 2}(\nu)\right]
\end{aligned}
$$

and

$$
\begin{aligned}
& {\left[y_{1}(\nu), y_{2}(\nu)\right]} \\
& =\sqrt{P_{1}} \gamma_{10} h_{10}(\nu)\left[c_{21}(\nu), c_{22}(\nu)\right]+\left[n_{1}(\nu), n_{2}(\nu)\right],
\end{aligned}
$$

where $n_{i}(\nu)$ and $n_{r i}(\nu)$ denote, respectively, the receiver noise at the destination and the relay at time slot $i$ within time block $\nu$.

At the second time block, $c_{11}(\nu)$ and $c_{12}(\nu)$ are transmitted from user 1's antenna, and $r_{1}(\nu)$ and $r_{2}(\nu)$ are relayed from user 2's antenna after the amplification. The received signal at the destination receiver becomes

$$
\begin{aligned}
& {\left[y_{3}(\nu), y_{4}(\nu)\right]} \\
& =\left[\sqrt{P_{1}} \gamma_{10} h_{10}(\nu), \sqrt{P_{2}} \gamma_{20} h_{20}(\nu)\right]\left[\begin{array}{cc}
\alpha c_{11}(\nu) & \alpha c_{12}(\nu) \\
\hat{c}_{21}(\nu) & \hat{c}_{22}(\nu)
\end{array}\right] \\
& +\left[n_{3}(\nu), n_{4}(\nu)\right] \\
& =\left[\sqrt{P_{1}} \gamma_{10} h_{10}(\nu), \sqrt{P_{1} P_{2}} \gamma_{20} \gamma_{12} h_{20}(\nu) h_{12}(\nu) g_{2}\right] \\
& \cdot\left[\begin{array}{cc}
\alpha c_{11}(\nu) & \alpha c_{12}(\nu) \\
c_{21}(\nu) & c_{22}(\nu)
\end{array}\right] \\
& +\sqrt{P_{2}} \gamma_{20} h_{20}(\nu) g_{2}\left[n_{r 1}(\nu), n_{r 2}(\nu)\right]+\left[n_{3}(\nu), n_{4}(\nu)\right] \\
& =\left[\sqrt{P_{1}} \gamma_{10} h_{10}(\nu), \sqrt{P_{2}} \gamma_{20} h_{20}(\nu) h_{12}(\nu) \tilde{g}_{2}\right] \\
& \text {. }\left[\begin{array}{rl}
\alpha c_{11}(\nu) & \alpha c_{12}(\nu) \\
c_{21}(\nu) & c_{22}(\nu)
\end{array}\right] \\
& +\sqrt{\frac{P_{2}}{P_{1}}} \frac{\gamma_{20}}{\gamma_{12}} h_{20} \tilde{g}_{2}\left[n_{r 1}(\nu), n_{r 2}(\nu)\right]+\left[n_{3}(\nu), n_{4}(\nu)\right],
\end{aligned}
$$

where

$$
\begin{aligned}
g_{2}^{2} & =\left\{E\left[\left|r_{1}(\nu)\right|^{2}+\left|r_{2}(\nu)\right|^{2}\right]\right\}^{-1} \\
& =\left\{E\left[P_{1} \gamma_{12}^{2}\left|h_{12}(\nu)\right|^{2}+n_{r 1}^{2}(\nu)+n_{r 2}^{2}(\nu)\right]\right\}^{-1} \\
& =\left[P_{1} \gamma_{12}^{2}+2 \sigma_{r n}^{2}\right]^{-1}=P_{1}^{-1} \gamma_{12}^{-2}\left[1+\frac{2 \sigma_{r n}^{2}}{P_{1} \gamma_{12}^{2}}\right]^{-1}
\end{aligned}
$$

is a power normalization factor such that the total energy transmitted from the relay terminal over the two symbols is $P_{2}$, and

$$
\tilde{g}_{2}^{2}=g_{2}^{2} P_{1} \gamma_{12}^{2}=\frac{P_{1} \gamma_{12}^{2}}{P_{1} \gamma_{12}^{2}+2 \sigma_{r n}^{2}} \leq 1
$$

is the power ratio at which the relay terminal transmits the desired signal in the presence of relay noise. In (17) and (18), $\sigma_{r n}^{2}=E\left[\left|n_{r 1}(\nu)\right|^{2}\right]=E\left[\left|n_{r 2}(\nu)\right|^{2}\right]$ is the variance of the relay noise.

Equations (14) - (17) can be combined into the following format

$$
\mathbf{y}(\nu)=\mathbf{h}(\nu) \mathbf{C}^{\prime}(\nu)+\underbrace{\mathbf{n}(\nu)+\sqrt{\frac{P_{2}}{P_{1}}} \frac{\gamma_{20}}{\gamma_{12}} h_{20} \tilde{g}_{2} \mathbf{n}_{r}(\nu)}_{\hat{\mathbf{n}}(\nu)},
$$

where

$$
\begin{aligned}
\mathbf{y}(\nu) & =\left[y_{1}(\nu), y_{2}(\nu), y_{3}(\nu), y_{4}(\nu)\right] \\
\mathbf{h}(\nu) & =\left[\gamma_{10} h_{10}(\nu), \gamma_{20} h_{20}(\nu) h_{12}(\nu) \tilde{g}_{2}\right] \\
\mathbf{n}(\nu) & =\left[n_{1}(\nu), n_{2}(\nu), n_{3}(\nu), n_{4}(\nu)\right] \\
\mathbf{n}_{r}(\nu) & =\left[0,0, n_{r 1}(\nu), n_{r 2}(\nu)\right]
\end{aligned}
$$


and

$\mathbf{C}^{\prime}(\nu)$
$=\left[\begin{array}{cccc}\sqrt{P_{1}} c_{21}(\nu) & \sqrt{P_{1}} c_{22}(\nu) & \alpha \sqrt{P_{1}} c_{11}(\nu) & \alpha \sqrt{P_{1}} c_{12}(\nu) \\ 0 & 0 & \sqrt{P_{2}} c_{21}(\nu) & \sqrt{P_{2}} c_{22}(\nu)\end{array}\right]$.

It is noted that, because of the relay noise, the average power that the relay terminal uses to transmit the useful information is $P_{2} \tilde{g}_{2}^{2} / 2$ for each symbol, whereas the average power transmitted from the relay terminal is $P_{2} / 2$ per symbol.

Similar to (19), for time block $\nu-1$, we have

$$
\begin{aligned}
\mathbf{y}(\nu-1)= & \mathbf{h}(\nu-1) \mathbf{C}^{\prime}(\nu-1)+\mathbf{n}(\nu-1) \\
& +\sqrt{\frac{P_{2}}{P_{1}}} \frac{\gamma_{20}}{\gamma_{12}} h_{20} \tilde{g}_{2} \mathbf{n}_{r}(\nu-1) \\
= & \mathbf{h}(\nu) \mathbf{C}^{\prime}(\nu-1)+\mathbf{n}(\nu-1) \\
& +\sqrt{\frac{P_{2}}{P_{1}}} \frac{\gamma_{20}}{\gamma_{12}} h_{20} \tilde{g}_{2} \mathbf{n}_{r}(\nu-1)
\end{aligned}
$$

where again $\mathbf{h}(\nu)=\mathbf{h}(\nu-1)$ is assumed. With the noise components considered, $\mathbf{y}(\nu)$ and $\mathbf{y}(\nu-1)$ are now related through the following relationship

$$
\mathbf{y}(\nu)=\mathbf{y}(\nu-1) \mathbf{G}^{\prime}(\nu)+\tilde{\mathbf{n}}(\nu)
$$

where

$$
\begin{aligned}
\tilde{\mathbf{n}}(\nu) & =\left[\mathbf{n}(\nu)+\sqrt{\frac{P_{2}}{P_{1}}} \frac{\gamma_{20}}{\gamma_{12}} h_{20} \tilde{g}_{2} \mathbf{n}_{r}(\nu)\right] \\
& -\left[\mathbf{n}(\nu-1)+\sqrt{\frac{P_{2}}{P_{1}}} \frac{\gamma_{20}}{\gamma_{12}} h_{20} \tilde{g}_{2} \mathbf{n}_{r}(\nu-1)\right] \mathbf{G}^{\prime}(\nu) .
\end{aligned}
$$

Because $\mathbf{G}^{\prime}(\nu)$ is unitary, it is evident from the above equation that the differential decoding doubles the noise power or, equivalently, reduces the SNR by $3 \mathrm{~dB}$.

\section{B. Maximum Likelihood Detection}

The covariance matrix of the combined noise vector $\tilde{\mathbf{n}}(\nu)$ is obtained as

$$
\begin{aligned}
\mathbf{R}_{\tilde{\mathbf{n}}} & =E\left[\tilde{\mathbf{n}}^{H}(\nu) \tilde{\mathbf{n}}(\nu)\right] \\
& =2 \sigma_{n}^{2}\left[\mathbf{I}_{4}+\frac{P_{2}}{P_{1}} \frac{\gamma_{20}^{2}}{\gamma_{12}^{2}} \frac{\sigma_{r n}^{2}}{\sigma_{n}^{2}} \tilde{g}_{2}^{2} \mathbf{D}_{2}\right]=\sigma_{n}^{2} \Delta^{2}
\end{aligned}
$$

where $\sigma_{n}^{2}=E\left[\left|n_{1}(\nu)\right|^{2}\right]=E\left[\left|n_{2}(\nu)\right|^{2}\right]$ is the variance of the destination receiver noise, $\mathbf{D}_{2}=\operatorname{diag}[0,0,1,1]$, and

$$
\begin{aligned}
\Delta^{2} & =2\left[\mathbf{I}_{4}+\frac{P_{2}}{P_{1}} \frac{\gamma_{20}^{2}}{\gamma_{12}^{2}} \frac{\sigma_{r n}^{2}}{\sigma_{n}^{2}} \tilde{g}_{2}^{2} \mathbf{D}_{2}\right] \\
& =2\left[\mathbf{I}_{4}+\frac{P_{2} \gamma_{20}^{2} \sigma_{r n}^{2}}{\sigma_{n}^{2}\left(P_{1} \gamma_{12}^{2}+2 \sigma_{r n}^{2}\right)} \mathbf{D}_{2}\right]=2\left[\mathbf{I}_{4}+W \mathbf{D}_{2}\right]
\end{aligned}
$$

is a diagonal matrix implicitly defined in the above equation, where

$$
W=\frac{P_{2} \gamma_{20}^{2} \sigma_{r n}^{2}}{\sigma_{n}^{2}\left(P_{1} \gamma_{12}^{2}+2 \sigma_{r n}^{2}\right)} \text {. }
$$

The maximum likelihood detection results in the estimation of $\mathbf{G}(\nu)$ as

$$
\begin{aligned}
& \hat{\mathbf{G}}(\nu)= \arg \min _{\mathbf{G}} \operatorname{tr}\left\{\left[\mathbf{y}(\nu) \Delta^{-1}-\mathbf{y}(\nu-1)\left(\mathbf{I}_{2} \otimes \mathbf{G}\right) \Delta^{-1}\right]\right. \\
& \cdot\left[\mathbf{y}(\nu) \Delta^{-1}-\mathbf{y}(\nu-1)\left(\mathbf{I}_{2} \otimes \mathbf{G}\right) \Delta^{-1}\right] \\
&=\arg \min _{\mathbf{G}} \operatorname{tr}\left\{\left[\mathbf{y}(\nu)-\mathbf{y}(\nu-1)\left(\mathbf{I}_{2} \otimes \mathbf{G}\right)\right]\right. \\
&\left.\cdot \Delta^{-2}\left[\mathbf{y}(\nu)-\mathbf{y}(\nu-1)\left(\mathbf{I}_{2} \otimes \mathbf{G}\right)\right]^{H}\right\},
\end{aligned}
$$

where "tr" denotes the trace of a matrix.

\section{Performance Analysis}

While the accurate performance of a differentially coded system requires the consideration of the quadratic receiving structure [22], [23], [26], it can be well approximated in high SNR situations by using an equivalent coherent receiver model (22) with $\mathbf{y}(\nu-1)$ acting as a known channel vector and $\tilde{\mathbf{n}}(\nu)$ as the equivalent noise vector of enhanced power [25].

From (24), it is clear that the variance of the combined noise term during the last two symbols is larger than that during the first two symbols. Assume that the covariance matrix $\mathbf{R}_{\tilde{\mathbf{n}}}=$ $\sigma_{n}^{2} \Delta^{2}$ can be estimated at the destination receiver. Then, right multiplying $\Delta^{-1}$ to (22) results in

$$
\mathbf{y}(\nu) \Delta^{-1}=\mathbf{y}(\nu-1) \mathbf{G}^{\prime}(\nu) \Delta^{-1}+\tilde{\mathbf{n}}(\nu) \Delta^{-1}
$$

or

$$
\mathbf{y}^{\prime}(\nu)=\mathbf{y}(\nu-1) \tilde{\mathbf{G}}(\nu)+\mathbf{n}^{\prime}(\nu)
$$

where $\mathbf{y}^{\prime}(\nu)=\mathbf{y}(\nu) \Delta^{-1}, \tilde{\mathbf{G}}(\nu)=\mathbf{G}^{\prime}(\nu) \Delta^{-1}$, and $\mathbf{n}^{\prime}(\nu)=$ $\tilde{\mathbf{n}}(\nu) \Delta^{-1}$ has a covariance matrix $\sigma_{n}^{2} \mathbf{I}_{4}$. Multiplying $\mathbf{y}(\nu)$ by $\Delta^{-1}$ amounts to adjusting the signal strength at the receiver so that the noise power of the four symbols becomes equal. It does not affect the total power transmitted at the source and relay terminals, and the cordword error probability as well.

From (28) and (29), it is seen that the detection problem becomes equivalent to finding unknown unitary codeword $\mathbf{G}^{\prime}(\nu)$ in known equivalent channel vector $\mathbf{y}(\nu-1)$, known noise covariance matrix $\sigma_{n}^{2} \Delta^{2}$, and independent and temporally white noise components. From the above two equations, we can derive the pairwise codeword error probability (CER), i.e., the probability of transmitting $\mathbf{G}^{\prime}=\operatorname{diag}[\mathbf{G}, \mathbf{G}]$ and deciding in favor of another $\mathbf{E}^{\prime}=\operatorname{diag}[\mathbf{E}, \mathbf{E}]$ at the detector, conditioned by the equivalent channel vector $\mathbf{y}(\nu-1)$. The CER is given by

$$
P\left(\mathbf{G}^{\prime} \rightarrow \mathbf{E}^{\prime} \mid \mathbf{y}(\nu-1)\right)=Q\left(\sqrt{d^{2}(\tilde{\mathbf{G}}, \tilde{\mathbf{E}}) /\left(2 \sigma_{n}^{2}\right)}\right),
$$

where $d^{2}(\tilde{\mathbf{G}}, \tilde{\mathbf{E}})$ is the distance between the received signals corresponding to respective codewords $\tilde{\mathbf{G}}(\nu)$ and $\tilde{\mathbf{E}}(\nu)$ and is expressed as

$$
d^{2}(\tilde{\mathbf{G}}, \tilde{\mathbf{E}})=\mathbf{y}(\nu-1) \mathbf{A} \mathbf{y}^{H}(\nu-1)
$$

$$
\begin{aligned}
\mathbf{A} & =(\tilde{\mathbf{G}}(\nu)-\tilde{\mathbf{E}}(\nu))(\tilde{\mathbf{G}}(\nu)-\tilde{\mathbf{E}}(\nu))^{H} \\
& =\left(\mathbf{G}^{\prime}(\nu)-\mathbf{E}^{\prime}(\nu)\right) \Delta^{-2}\left(\mathbf{G}^{\prime}(\nu)-\mathbf{E}^{\prime}(\nu)\right)^{H}
\end{aligned}
$$


In calculating the distance of (31), we make the following approximation $\mathbf{y}(\nu) \approx \mathbf{h}(\nu) \mathbf{C}^{\prime}(\nu)$ for moderate or high SNR scenarios. Then, (31) becomes

$$
\begin{aligned}
d^{2}(\tilde{\mathbf{G}}, \tilde{\mathbf{E}}) & =\mathbf{h}(\nu-1) \mathbf{C}^{\prime}(\nu-1) \mathbf{A}\left(\mathbf{C}^{\prime}(\nu-1)\right)^{H} \mathbf{h}^{H}(\nu-1) \\
& =\tilde{\mathbf{h}}(\nu) \boldsymbol{\Sigma}_{\mathbf{h}} \mathbf{C}^{\prime}(\nu-1) \mathbf{A}\left(\mathbf{C}^{\prime}(\nu-1)\right)^{H} \boldsymbol{\Sigma}_{\mathbf{h}} \tilde{\mathbf{h}}^{H}(\nu),
\end{aligned}
$$

where

$$
\boldsymbol{\Sigma}_{\mathbf{h}}=\operatorname{diag}\left[\sigma_{1}, \sigma_{2}\right]=\operatorname{diag}\left[\gamma_{10}, \gamma_{20} \tilde{g}_{2}\right]
$$

and

$$
\tilde{\mathbf{h}}(\nu)=\mathbf{h}(\nu) \boldsymbol{\Sigma}_{\mathbf{h}}^{-1 / 2}=\left[h_{10}(\nu), h_{12}(\nu) h_{20}(\nu)\right]
$$

is the normalized channel vector. For simplicity of the performance analysis, we assume the relay channel $h_{12}(\nu)$ is dominated by the time-invariant component (e.g., source terminal and relay terminal have a clear line-of-sight and scattering is relatively weak) and, therefore, can be approximated by a unit value. In addition, $h_{10}(\nu)$ and $h_{20}(\nu)$ are assumed to be uncorrelated complex Gaussian processes. In this case, $\tilde{\mathbf{h}}(\nu)=\left[h_{10}(\nu), h_{20}(\nu)\right] \sim \mathcal{C} N\left(0, \mathbf{I}_{2}\right)$.

In the underlying situation, the average power per symbol is $E_{s}=P / 4$ (i.e., the total energy of a DSTC codeword is $P$ ). To clearly show the relationship between the pairwise CER and the input SNR, we rewrite (30) as

$$
\begin{aligned}
P\left(\mathbf{G}^{\prime} \rightarrow \mathbf{E}^{\prime} \mid \mathbf{y}(\nu-1)\right) & =Q\left(\sqrt{\frac{d^{2}(\tilde{\mathbf{G}}, \tilde{\mathbf{E}})}{P} \frac{2 E_{s}}{\sigma_{n}^{2}}}\right) \\
& \leq \exp \left[\frac{-d^{2}(\tilde{\mathbf{G}}, \tilde{\mathbf{E}})}{P} \frac{E_{s}}{\sigma_{n}^{2}}\right],
\end{aligned}
$$

where the inequality is the Chernoff bound [2]. Because $\mathbf{y}(\nu)$ is approximated as a linear combination of i.i.d. channels $\tilde{\mathbf{h}}(\nu)$ and, therefore, constitute a set of dependent channel coefficients, averaging the above bound with respect to $\mathbf{y}(\nu-$ 1) results in [2]

$$
P\left(\mathbf{G}^{\prime} \rightarrow \mathbf{E}^{\prime}\right) \leq \prod_{i=1}^{2}\left(1+\frac{E_{s}}{\sigma_{n}^{2}} \frac{d_{i}}{P}\right)^{-1},
$$

where $d_{1}$ and $d_{2}$ are the eigenvalues of the following $2 \times 2$ matrix

$$
\mathbf{K}=\boldsymbol{\Sigma}_{\mathbf{h}} \mathbf{C}^{\prime}(\nu-1) \mathbf{A}\left(\mathbf{C}^{\prime}(\nu-1)\right)^{H} \boldsymbol{\Sigma}_{\mathbf{h}} .
$$

In the following, we consider two different cases. In the first case, matrix $\mathbf{K}$ is full rank, whereas in the second case, matrix $\mathbf{K}$ is rank one.

\section{Case I: Full Rank Channel Environment}

When $E_{s} d_{i} /\left(P \sigma_{n}^{2}\right) \gg 1$ for $i=1,2$, the CER can be approximated as

$$
\begin{aligned}
P\left(\mathbf{G}^{\prime} \rightarrow \mathbf{E}^{\prime}\right) & \leq \prod_{i=1}^{2}\left(1+\frac{E_{s}}{\sigma_{n}^{2}} \frac{d_{i}}{P}\right)^{-1} \\
& \leq\left(\frac{E_{s}}{\sigma_{n}^{2}}\right)^{-2} \prod_{i=1}^{2}\left(\frac{d_{i}}{P}\right)^{-1} \\
& =\left(\frac{E_{s}}{4 \sigma_{n}^{2}}\right)^{-2} \prod_{i=1}^{2}\left(\frac{4 d_{i}}{P}\right)^{-1} .
\end{aligned}
$$

The term $\left(\frac{E_{s}}{4 \sigma_{n}^{2}}\right)^{-2}$ determines the diversity gain which indicates the slope at which the BER changes with the input SNR, whereas the term $\prod_{i=1}^{2}\left(\frac{4 d_{i}}{P}\right)$ is a constant gain in controlling the BER and is referred to as the diversity product. In this case, the diversity gain of the system is two. Using the above results, it is seen that the diversity product is determined by the minimum product of $d_{1} d_{2}$, i.e., the minimum determinant of $\mathbf{K}$. It can be shown that (refer to the Appendix)

$$
\begin{aligned}
\min \operatorname{det}(\mathbf{K}) & \frac{1}{4(1+W)^{2}}\left|\operatorname{det}\left(\boldsymbol{\Sigma}_{\mathbf{h}}\right) \operatorname{det}\left(\mathbf{C}_{2}\right)\right|^{2}(\min |\operatorname{det}(\mathbf{G}-\mathbf{E})|)^{2} \\
& +\frac{P_{1} P_{2} \gamma_{10}^{2} \gamma_{20}^{2} \tilde{g}_{2}^{2}}{4(1+W)}\left(\min \mathbf{c}_{1}(\mathbf{G}-\mathbf{E})(\mathbf{G}-\mathbf{E})^{H} \mathbf{c}_{1}^{H}\right)^{2} \\
= & \frac{\alpha^{2} P_{1} P_{2} \gamma_{10}^{2} \gamma_{20}^{2} \tilde{g}_{2}^{2}}{4(1+W)^{2}}(\min |\operatorname{det}(\mathbf{G}-\mathbf{E})|)^{2} \\
& +\frac{P_{1} P_{2} \gamma_{10}^{2} \gamma_{20}^{2} \tilde{g}_{2}^{2}}{4(1+W)}\left(\min \mathbf{c}_{1}(\mathbf{G}-\mathbf{E})(\mathbf{G}-\mathbf{E})^{H} \mathbf{c}_{1}^{H}\right)^{2},
\end{aligned}
$$

where $W$ is defined in (26),

$$
\mathbf{c}_{1}=\left[c_{21}, c_{22}\right],
$$

and

$$
\begin{aligned}
\mathbf{C}_{2} & =\left[\begin{array}{cc}
\alpha \sqrt{P_{1}} & 0 \\
0 & \sqrt{P_{2}}
\end{array}\right]\left[\begin{array}{ll}
c_{11} & c_{12} \\
c_{21} & c_{22}
\end{array}\right] \\
& =\left[\begin{array}{cc}
\alpha \sqrt{P_{1}} c_{11} & \alpha \sqrt{P_{1}} c_{12} \\
\sqrt{P_{2}} c_{21} & \sqrt{P_{2}} c_{22}
\end{array}\right] .
\end{aligned}
$$

The value of $\mathbf{c}_{1}(\mathbf{G}-\mathbf{E})(\mathbf{G}-\mathbf{E})^{H} \mathbf{c}_{1}^{H}$ is lower bounded by $\lambda_{\min }\left[(\mathbf{G}-\mathbf{E})(\mathbf{G}-\mathbf{E})^{H}\right]$, which is the minimum value of the smallest eigenvalues with respect to all the combinations of $\mathbf{G}$ and $\mathbf{E}$. That is,

$$
\mathbf{c}_{1}(\mathbf{G}-\mathbf{E})(\mathbf{G}-\mathbf{E})^{H} \mathbf{c}_{1}^{H} \geq \lambda_{\min }\left[(\mathbf{G}-\mathbf{E})(\mathbf{G}-\mathbf{E})^{H}\right] .
$$

Define

$$
\beta=\frac{\lambda_{\min }\left[(\mathbf{G}-\mathbf{E})(\mathbf{G}-\mathbf{E})^{H}\right]}{\min |\operatorname{det}(\mathbf{G}-\mathbf{E})|}
$$

as a factor determined by the used differential code. Then, the diversity product is lower bounded by

$$
\begin{aligned}
\min \operatorname{det}(\mathbf{K})\left(\frac{4}{P}\right)^{2} \geq \frac{4 P_{1} P_{2} \gamma_{10}^{2} \gamma_{20}^{2} \tilde{g}_{2}^{2}}{\left[\left(1+\alpha^{2}\right) P_{1}+P_{2}\right]^{2}} \\
. \frac{\left[\alpha^{2}+(1+W) \beta^{2}\right]}{(1+W)^{2}}(\min |\operatorname{det}[\mathbf{G}-\mathbf{E}]|)^{2} .
\end{aligned}
$$

Now we consider the maximization of (45) in terms of the power allocation, leading to the optimal selection of $P_{1}, P_{2}$, and $\alpha$. Because $(\min |\operatorname{det}[\mathbf{G}-\mathbf{E}]|)^{2}$ is independent of the power allocation, it suffices to consider only the following part of (45) for the purpose of optimization of the power allocation,

$$
Q_{1}=\frac{4 P_{1} P_{2} \gamma_{10}^{2} \gamma_{20}^{2} \tilde{g}_{2}^{2}}{\left[\left(1+\alpha^{2}\right) P_{1}+P_{2}\right]^{2}} \frac{\left[\alpha^{2}+(1+W) \beta^{2}\right]}{(1+W)^{2}} .
$$

By letting $\partial Q_{1} / \partial \alpha=0$, we get

$$
\begin{aligned}
\alpha_{\mathrm{opt}}^{2} & =\left[1-2(1+W) \beta^{2}+P_{2} / P_{1}\right]^{+} \\
& =\left[1-2\left(1+\frac{P_{2} \gamma_{20}^{2} \sigma_{r n}^{2}}{\sigma_{n}^{2}\left(P_{1} \gamma_{12}^{2}+2 \sigma_{r n}^{2}\right)}\right) \beta^{2}+\frac{P_{2}}{P_{1}}\right]^{+},
\end{aligned}
$$


where $[x]^{+}=\max (0, x)$ (This operator is used to ensure that $\alpha^{2}$ takes non-negative values). While the optimum power allocation between $P_{1}$ and $P_{2}$ can be obtained from the above expression, the analytic result is prohibitively complicated. However, the above results are helpful in determining the optimum power allocation. Specifically, when the interuser channel between the source and relay terminal has a good quality, i.e., $\gamma_{12}^{2}$ is large and $\sigma_{r n}^{2}$ is small, then $\tilde{g}^{2} \approx 1$ and $W \approx 0$, and $Q_{1}$ becomes

$$
Q_{1}^{\prime} \approx \frac{4 P_{1} P_{2} \gamma_{10}^{2} \gamma_{20}^{2}}{\left(1+\alpha^{2}\right) P_{1}+P_{2}}\left(\alpha^{2}+\beta^{2}\right)
$$

Letting $\partial Q_{1}^{\prime} / \partial P_{1}=0$ yields

$$
\left(1+\alpha^{2}\right) P_{1}=P_{2} \text {. }
$$

Substituting this result back to (48), we obtain

$$
Q_{1, \mathrm{opt}}^{\prime} \approx \frac{\gamma_{10}^{2} \gamma_{20}^{2}\left(\alpha^{2}+\beta^{2}\right)}{1+\alpha^{2}}
$$

In particular, when $\beta^{2}=1, Q_{1, \mathrm{opt}}^{\prime}$ is independent of $\alpha$, providing that (49) is satisfied. On the other hand, $\alpha^{2}$ assumes a large value when $\beta^{2}<1$, resulting in $P_{1} \ll P_{2}$ and $\alpha^{2} P_{1} \approx P_{2}$.

Therefore, the optimum value of $\alpha$ depends on $\beta$. That is, given the same environment, different differential codes do not necessarily allocate the power in the same manner. When the relay noise is not negligible, the optimal value of $Q_{1}^{\prime}$ is compromised by the relay noise and in general $\beta$-dependent. Therefore, a differential code with higher $\min |\operatorname{det}(\mathbf{G}-\mathbf{E})|$ does not necessarily imply a high diversity product in the underlying cooperative diversity applications.

It is clear that, to optimize the power allocation, the channel attenuation characteristics have to be known. While in this paper we assume that the exact CSI is unavailable at the transmitters and receivers, we maintain that it is often practical to assume the channel quality at both transmitters and receivers because the attenuation characteristics vary with time in a much slower manner. It is emphasized that such large-scale channel attenuation characteristics are not required in employing the proposed differential distributed space-time coding scheme, but a good estimation of such information can be used to improve the system performance.

\section{Case II: Rank 1 Channel Environment}

When $E_{s} d_{1} /\left(P \sigma_{n}^{2}\right) \gg 1$ whereas $E_{s} d_{2} /\left(P \sigma_{n}^{2}\right) \ll 1$, (39) is no longer applicable. It happens, for example, when either the direct link or the relay link is highly impairing. In this case, (37) becomes

$$
\begin{aligned}
P(\tilde{\mathbf{G}} \rightarrow \tilde{\mathbf{E}}) & \leq \prod_{i=1}^{2}\left(1+\frac{E_{s}}{\sigma_{n}^{2}} \frac{d_{i}}{P}\right)^{-1} \\
& \leq\left(\frac{E_{s}}{\sigma_{n}^{2}}\right)^{-1}\left(\frac{d_{1}}{P}\right)^{-1} \\
& =\left(\frac{E_{s}}{4 \sigma_{n}^{2}}\right)^{-1}\left(\frac{4 d_{1}}{P}\right)^{-1} .
\end{aligned}
$$

When the relay channel is not reliable, that is, either $\gamma_{20}$ or $\gamma_{12}$ is very small, or the relay noise $\sigma_{r n}^{2}$ is very large, it is obvious that the relay should not be used and the system degenerates to the non-cooperative system.
On the other hand, when the direct channel between the source and the destination is unreliable, i.e., $\gamma_{10} \approx 0$, the system becomes a pure relay structure. In this case, $\alpha$ should be chosen as 0 , and $\mathbf{K}$ defined in (38) becomes

$$
\mathbf{K}=\operatorname{diag}\left[0, \frac{P_{2} \gamma_{20}^{2} \tilde{g}_{2}^{2}}{2(1+W)} \mathbf{c}_{1}(\mathbf{G}-\mathbf{E})(\mathbf{G}-\mathbf{E})^{H} \mathbf{c}_{1}^{H}\right] .
$$

Using the results of (43) and (44), the minimum value of $4 d_{1} / P$ in (51) is obtained as

$$
\begin{aligned}
\min & \left(\frac{4 d_{1}}{P}\right) \\
& =\frac{1}{P} \frac{4 P_{2} \gamma_{20}^{2} \tilde{g}_{2}^{2}}{2(1+W)} \min \left[\mathbf{c}_{1}(\mathbf{G}-\mathbf{E})(\mathbf{G}-\mathbf{E})^{H} \mathbf{c}_{1}^{H}\right] \\
& \geq \frac{2 P_{1} P_{2} \gamma_{12}^{2} \gamma_{20}^{2}}{P_{1}+P_{2}} \frac{\sigma_{n}^{2} \beta \min |\operatorname{det}(\mathbf{G}-\mathbf{E})|}{\left[\sigma_{n}^{2}\left(P_{1} \gamma_{12}^{2}+2 \sigma_{r n}^{2}\right)+P_{2} \gamma_{20}^{2} \sigma_{r n}^{2}\right]} .
\end{aligned}
$$

Because $\beta \min |\operatorname{det}(\mathbf{G}-\mathbf{E})|$ is independent of the power allocation, we only consider the following term for the optimization of the power allocation,

$$
Q_{2}=\frac{2 P_{1} P_{2} \gamma_{12}^{2} \gamma_{20}^{2}}{P_{1}+P_{2}} \frac{\sigma_{n}^{2}}{\left[\sigma_{n}^{2}\left(P_{1} \gamma_{12}^{2}+2 \sigma_{r n}^{2}\right)+P_{2} \gamma_{20}^{2} \sigma_{r n}^{2}\right]} .
$$

Then, $\partial Q_{2} / \partial P_{1}=0$ results in

$$
P_{2}=\left[\sqrt{\sigma_{n}^{2} \sigma_{r n}^{2}+\gamma_{20}^{2} \gamma_{12}^{2} P_{1}^{2}}-\sigma_{n} \sigma_{r n}\right] \sigma_{n} /\left(\gamma_{20}^{2} \sigma_{r n}\right) \text {. }
$$

In particular, when we assume $P_{1} \gamma_{12} \gg 2 \sigma_{r n}^{2}$, that is, the interuser channel has a high quality and the input SNR at the relay terminal is high, the above result becomes

$$
P_{2} \gamma_{20} / \sigma_{n}=P_{1} \gamma_{12} / \sigma_{r n} .
$$

Therefore, the power at different relay portions should be assigned proportional to the propagation gain and inversely proportional to the standard deviation of the noise generated in each relay portion.

\section{Numerical Results}

In this section, we consider a simple situation where two users cooperate to transmit their respective information to the destination, as illustrated in Fig. 4. Two differential DSTC codes are considered. In Scheme 1, the differential DSTC code is constructed based on Alamouti's space-time code, where each symbol uses a QPSK signal constellation, results in $N=16$ codewords in the two time-slot space-time code. In Scheme 2, the differential DSTC is constructed based on the differential MIMO space-time code developed by Liang and Xia [27]. For the latter, the constellation size remains $N=16$. Unless otherwise specified, the power is optimally allocated by adjusting $P_{1}, P_{2}$, and $\alpha$ to maximize $Q_{1}$ in (46), provided that the average power per symbol is unity.

\section{A. Comparison with Co-located MIMO Systems}

In the first set of simulations, we compare the CER performance of the proposed cooperative network with a MIMO system consisting of two co-located antennas. The two antennas in the MIMO system are assumed to have independent Rayleigh channels to the destination with unit variance. For 


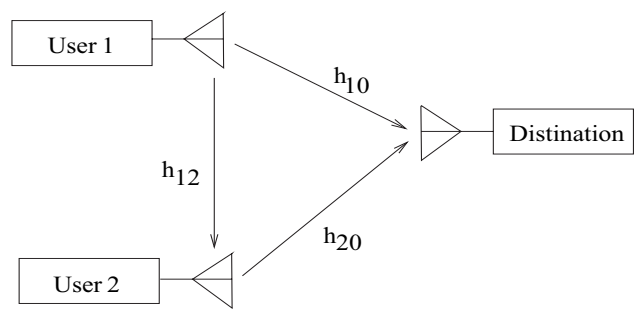

Fig. 4. Two-user system diagram.

the cooperative system, we assume $\gamma_{10}=\gamma_{20}=1$ and $\gamma_{12}=\sqrt{125}$, which corresponds to the situation that the interuser distance between the source and relay terminals is one-fifth of the distance between the source terminal and the destination, and the power attenuation exponential is 3 . In this case, the channels to the destination terminal, i.e., $h_{10}(\nu)$ and $h_{20}(\nu)$, are assumed Rayleigh faded, whereas the interuser channel is considered non-fading, i.e., $h_{12}(\nu)=1$.

Fig. 5 compares the performance of the cooperative diversity system and the corresponding MIMO system. The SNR is defined as the reciprocal of the receiver noise power, as the transmit power is normalized to unity per symbol period. The relay receiver and the destination terminal receiver have the same noise power. It is seen that, the cooperative diversity system achieves the same diversity gain, and there is a performance degradation due to the relay noise in the cooperative diversity system. The performance degradation is very small when the channel quality between the source and relay is reasonably good.

We notice that, however, the spectrum efficiency of the cooperative network is half of the corresponding MIMO system because of the requirement of the broadcast phase.

\section{B. Significance of Power Allocation}

In the second set of simulations, we consider a scenario where $\gamma_{10}=1$ and $\gamma_{12}=\gamma_{20}=\sqrt{8}$, which matches the situation that the relay terminal is in the mid-way between the source terminal and the destination. The power attenuation exponential remains to be 3 . All the channels are considered Rayleigh faded. To examine the effect of power allocation, Fig. 6 compares the CER based on optimal power allocation and a fix power allocation with $P_{1}=1, P_{2}=2$, and $\alpha=1$. Both cases have the same total energy of four over the foursymbol interval. It is seen that, compared to the optimal power allocation, the fixed power allocation yields an SNR loss of about $1.5 \mathrm{~dB}$ when the Alamouti code is used, and up to about $1 \mathrm{~dB}$ when the Liang-Xia code is used.

\section{Differential and Coherent Detections}

In the third set of simulations, we compare the differential and coherent detections, and the parameters are identical to the second set. In Fig. 7, we compare the CER performance using differential detection and coherent detection, where both differential DSTC coding schemes are considered. The respective optimum power allocation is applied. It is evident that, irrespective to the DSTC coding scheme used, the difference between the differential detection and coherent detection is merely the $3 \mathrm{~dB}$ noise amplification.

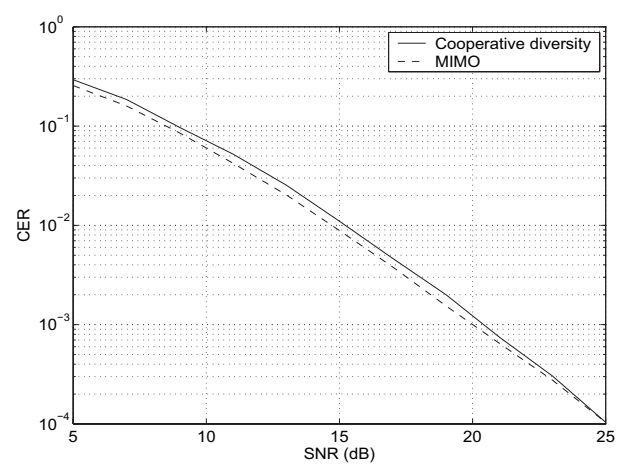

(a) Alamouti code

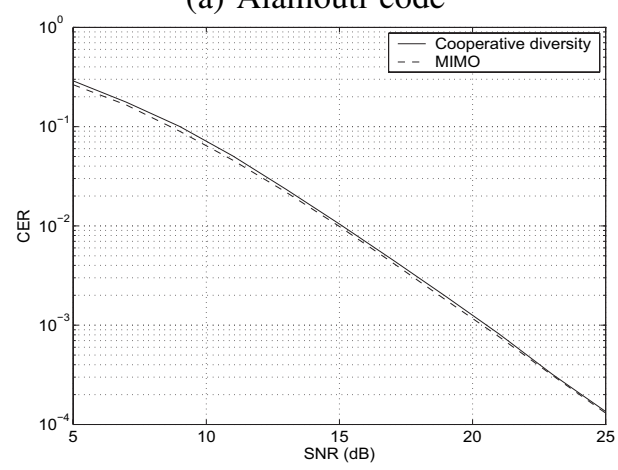

(b) Liang-Xia code

Fig. 5. Comparison of the CER performance between MIMO and cooperative diversity systems.

\section{Comparison with Existing Method}

The fourth set of simulations compares the performance of the proposed method with a relay system using differential modulation [32]. The parameter setting is similar to that used at [32], i.e., $\gamma_{10}=\gamma_{20}=\gamma_{12}=1$. However, we use QPSK modulation in Zhao and Li's method for easy and fair comparison because the Liang-Xia code is constructed using complex constellations. The simulation results in Fig. 8 show that the proposed method slightly outperforms the method developed by Zhao and Li. Their method is equivalent to a diagonal codeword with equal power allocation, whereas the method we proposed in this paper uses more general codeword structure with flexible power allocation.

\section{CONCLUSIONS}

A novel space-time cooperation protocol has been developed for effective cooperative diversity operation when the channel state information (CSI) is unavailable at the receivers. It has been shown that the proposed differential space-time cooperation scheme is effective, and the performance loss due to differential detection is limited to $3 \mathrm{~dB}$ of noise enhancement compared to coherent detection. We have analyzed the pairwise codeword error probability performance of the cooperative diversity system. Based on this result, the optimization of the power allocation over the source and relay terminals are considered and the results provided useful guidelines in system designs. 


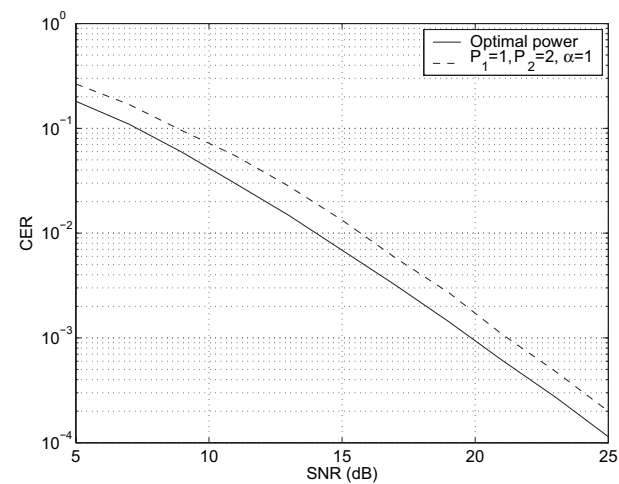

(a) Alamouti code

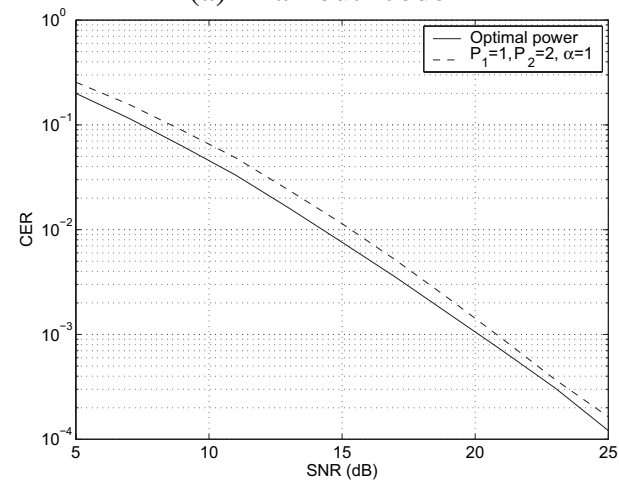

(b) Liang-Xia code

Fig. 6. Comparison of the CER performance for different power allocations.

\section{APPENDIX: DERIVATION OF EQUATION (40)}

We first consider the minimum determinant of $\mathbf{C}^{\prime}(\nu-$ 1) $\mathbf{A}\left(\mathbf{C}^{\prime}(\nu-1)\right)^{H}$. For notation simplicity, we omit $(\nu-1)$ in the rest of this appendix. Partition matrix $\mathbf{C}^{\prime}$ as

$$
\mathbf{C}^{\prime}=\left[\begin{array}{cc}
\sqrt{P_{1}} \mathbf{c}_{1} & \mathbf{C}_{2} \\
\mathbf{0} &
\end{array}\right]
$$

where $\mathbf{c}_{1}$ and $\mathbf{C}_{2}$ are defined in (42), and $\mathbf{0}=[0,0]$. Also denote $\mathbf{O}$ as the $2 \times 2$ zero matrix. Then we have

$$
\begin{aligned}
& \mathbf{C}^{\prime} \mathbf{A}\left(\mathbf{C}^{\prime}\right)^{H}=\left[\begin{array}{cc}
\sqrt{P_{1}} \mathbf{c}_{1} & : \mathbf{C}_{2} \\
\mathbf{0} &
\end{array}\right]\left[\begin{array}{cc}
\mathbf{G}-\mathbf{E} & \mathbf{O} \\
\mathbf{O} & \mathbf{G}-\mathbf{E}
\end{array}\right] \\
& \cdot\left[\begin{array}{cc}
\mathbf{I}_{2} / 2 & \mathbf{O} \\
\mathbf{O} & {[2(1+W)]^{-1} \mathbf{I}_{2}}
\end{array}\right] \\
& \cdot\left[\begin{array}{cc}
(\mathbf{G}-\mathbf{E})^{H} & \mathbf{O} \\
\mathbf{O} & (\mathbf{G}-\mathbf{E})^{H}
\end{array}\right]\left[\begin{array}{c}
\sqrt{P_{1}} \mathbf{c}_{1}^{H} \\
\mathbf{C}_{2}^{H} \\
\mathbf{C}_{2}^{H}
\end{array}\right] \\
& =\frac{1}{2}\left[\begin{array}{cc}
P_{1} \mathbf{c}_{1}(\mathbf{G}-\mathbf{E})(\mathbf{G}-\mathbf{E})^{H} \mathbf{c}_{1}^{H} & 0 \\
0 & 0
\end{array}\right] \\
& +\frac{1}{2(1+W)}\left[\mathbf{C}_{2}(\mathbf{G}-\mathbf{E})(\mathbf{G}-\mathbf{E})^{H} \mathbf{C}_{2}^{H}\right] .
\end{aligned}
$$

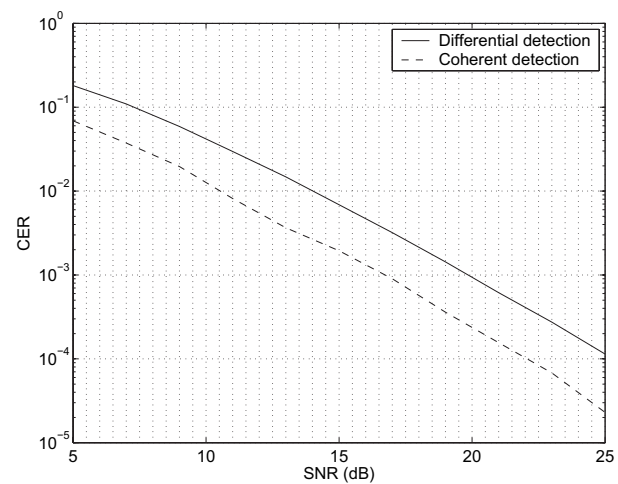

(a) Alamouti code

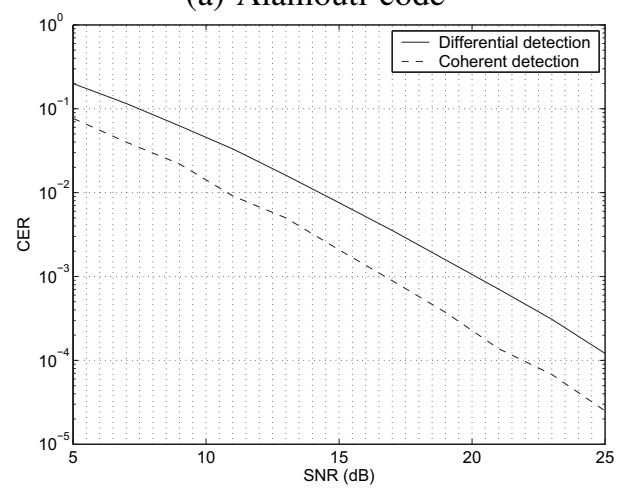

(b) Liang-Xia code

Fig. 7. Comparison of the CER performance between differential detection and coherent detection.

Because

$$
\begin{aligned}
\operatorname{det}\left(\left[\begin{array}{cc}
z_{0} & 0 \\
0 & 0
\end{array}\right]+\left[\begin{array}{cc}
z_{1} & z_{2} \\
z_{3} & z_{4}
\end{array}\right]\right) & =\operatorname{det}\left[\begin{array}{cc}
z_{1}+z_{0} & z_{2} \\
z_{3} & z_{4}
\end{array}\right] \\
& =\operatorname{det}\left[\begin{array}{cc}
z_{1} & z_{2} \\
z_{3} & z_{4}
\end{array}\right]+z_{0} z_{4}
\end{aligned}
$$

and the last (right-bottom) element of $\mathbf{C}_{2}(\mathbf{G}-\mathbf{E})(\mathbf{G}-$ $\mathbf{E})^{H} \mathbf{C}_{2}^{H}$ equals to $P_{2} \mathbf{c}_{1}(\mathbf{G}-\mathbf{E})(\mathbf{G}-\mathbf{E}){ }^{H} \mathbf{c}_{1}^{H}$, then, we have

$$
\begin{array}{r}
\operatorname{det}\left(\mathbf{C}^{\prime} \mathbf{A}\left(\mathbf{C}^{\prime}\right)^{H}\right)=\frac{1}{4(1+W)^{2}}\left|\operatorname{det}\left(\mathbf{C}_{2}\right) \operatorname{det}(\mathbf{G}-\mathbf{E})\right|^{2} \\
+\frac{1}{4(1+W)} P_{1} P_{2}\left[\mathbf{c}_{1}(\mathbf{G}-\mathbf{E})(\mathbf{G}-\mathbf{E})^{H} \mathbf{c}_{1}^{H}\right]^{2} .
\end{array}
$$

Noting the fact that

$$
\begin{aligned}
\left|\operatorname{det}\left(\mathbf{C}_{2}\right)\right|^{2} & =\operatorname{det}\left(\mathbf{C}_{2} \mathbf{C}_{2}^{H}\right) \\
& =\operatorname{det}\left\{\left[\begin{array}{cc}
\alpha \sqrt{P_{1}} & 0 \\
0 & \sqrt{P_{2}}
\end{array}\right] \mathbf{C C}^{H}\left[\begin{array}{cc}
\alpha \sqrt{P_{1}} & 0 \\
0 & \sqrt{P_{2}}
\end{array}\right]\right\} \\
& =\alpha^{2} P_{1} P_{2},
\end{aligned}
$$

equation (40) follows straightforwardly from (A.4).

\section{ACKNOWLEDGMENT}

The authors wish to thank the anonymous reviewers for their helpful comments and their thoughtful reviews. They greatly improved the qualities of the paper. 


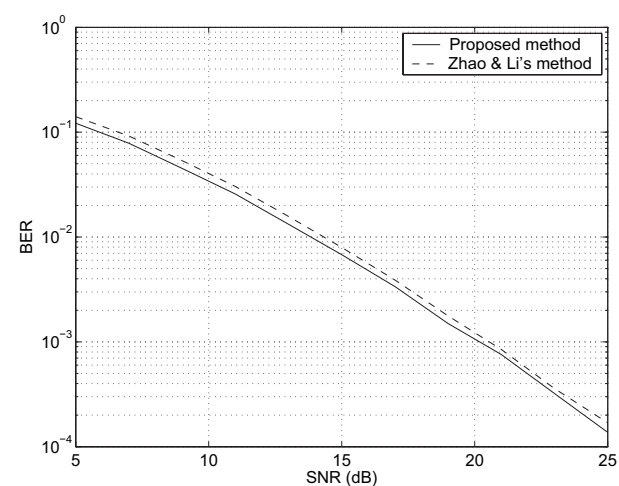

(a) Alamouti code

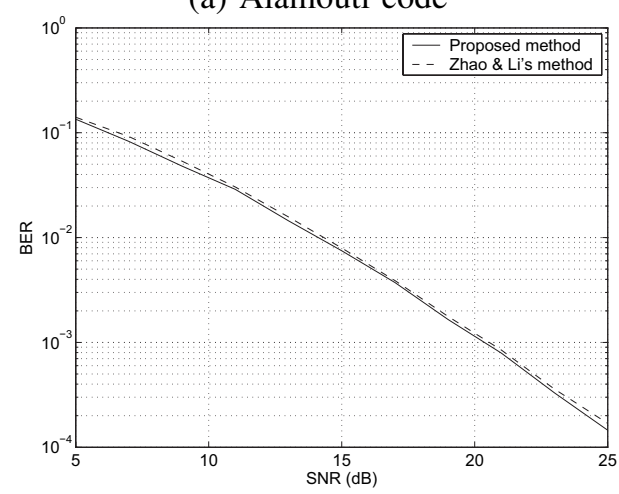

(b) Liang-Xia code

Fig. 8. Comparison of the BER performance between the proposed method and the method developed by Zhao and Li [32].

\section{REFERENCES}

[1] G. J. Foschini and M. J. Gans, "On limits of wireless communications in a fading environment when using multiple antennas," Wireless Pers. Commun., vol. 6, no. 3, pp. 311-335, Mar. 1998.

[2] V. Tarokh, H. Jafarkhani, and A. R. Calderbank, "Space-time block codes from orthogonal designs," IEEE Trans. Inf. Theory, vol. 45, no. 5, July 1999 .

[3] S. M. Alamouti, "A simple transmitter diversity scheme for wireless communications," IEEE J. Sel. Areas Commun., vol. 16, pp. 1451-1458, Oct. 1998.

[4] A. F. Naguib, N. Seshadri, A. R. Calderbank, "Increasing data rate over wireless channels: space-time coding and signal processing for high data rate wireless communications," IEEE Signal Process. Mag., vol. 17, no. 3, pp. 76-92, May 2000.

[5] A. Sendonaris, E. Erkip, and B. Aazhang, "Increasing uplink capacity via user cooperation diversity," in Proc. IEEE Int. Symp. Info. Theory 1998, p. 156.

[6] A. Sendonaris, E. Erkip, and B. Aazhang, "User cooperative diversity - Part I and Part II," IEEE Trans. Commun., vol. 51, no. 11, pp. 1927-1948, Nov. 2003.

[7] J. N. Laneman, D. N. C. Tse, and G. W. Wornell, "Cooperative diversity in wireless networks: effective protocols and outrage behavior," IEEE Trans. Inf. Theory, accepted for publication.

[8] J. N. Laneman and G. W. Wornell, "Distributed space-time coded protocols for exploiting cooperative diversity in wireless networks," IEEE Trans. Inf. Theory, vol. 49, no. 10, pp. 24152425, Oct. 2003.

[9] A. F. Dana and B. Hassibi, "On the power effeciency of sensory and ad-hoc wireless networks," submitted to IEEE Trans. Inf. Theory.

[10] M. Dohler, E. Lefranc, H. Aghvami, "Space-time block codes for virtual antenna arrays," in Proc. IEEE Int. Symp. Personal, Indoor and Mobile Radio Communications 2002, pp. 414-417.
[11] P. A. Anghel, G. Leus, and M. Kaveh, "Multi-user space-time coding in a cooperative networks," in Proc. IEEE ICASSP 2003.

[12] Y. Hua, Y. Mei, and Y. Chang, "Parallel wireless mobile relays with space-time modulations," in Proc. IEEE Workshop on Statistical Signal Processing 2003.

[13] A. Stefanov and E. Erkip, "On the performance analysis of cooperative space-time coded systems," in Proc. IEEE Wireless and Communications and Networking Conf. 2003.

[14] A. Stefanov and E. Erkip, "Cooperative space-time coding for wireless networks," in Proc. IEEE Information Theory Workshop 2003.

[15] A. Stefanov and E. Erkip, "Cooperative coding for wireless networks," IEEE Trans. Commun., vol. 52, no. 9, pp. 1470-1476, Sept. 2004.

[16] I. Hammerstroem, M. Kuhn, B. Rankov, A. Wittneben, "Spacetime processing for cooperative relay networks," in Proc. IEEE Vehicular Technology Conf. 2003.

[17] R. U. Nabar and H. Bölcskei, "Space-time signal design for fading relay channels," in Proc. GLOBECOM 2003, vol. 4, pp. 1952-1956.

[18] R. U. Nabar, F. W. Kneubühler, and H. Bölcskei, "Space-time signal design for fading relay channels," in Proc. GLOBECOM 2003.

[19] M. Janani, A. Hedayat, T. E. Hunter, and A. Nosratinia, "Coded cooperation in wireless communications: space-time transmission and iterative decoding," IEEE Trans. Signal Process., vol. 52, no. 2, pp. 362-371, Feb. 2004.

[20] Y. Jing and B. Hassibi, "Distributed space-time coding in wireless relay networks-Part I: basic diversity results," Submitted to IEEE Trans. Wireless Commun..

[21] Y. Jing and B. Hassibi, "Distributed space-time coding in wireless relay networks-Part II: tighter upper bounds and a more general case," Submitted to IEEE Trans. Wireless Commun..

[22] B. L. Hughes, "Differential space-time modulation," IEEE Trans. Inf. Theory, vol. 46, pp. 2567-2578, Nov. 2000.

[23] B. M. Hochwald and T. L. Marzetta, "Unitary space-time modulation for multiple-antenna communications in Rayleigh flat fading," IEEE Trans. Inf. Theory, vol. 46, pp. 543-564, Mar. 2000.

[24] B. M. Hochwald and W. Sweldens, "Differential unitary spacetime modulation," IEEE Trans. Commun., vol. 48, pp. 2041-2052, Dec. 2000.

[25] V. Tarokh and H. Jafarkhani, "A differential detection scheme for transmit diversity," IEEE J. Sel. Areas Commun., vol. 18, pp. 1169-1174, July 2000.

[26] M. Berhler, and M. K. Varanasi, "Asymptotic error probability analysis of quadratic receivers in Rayleigh-fading channels with applications to unified analysis of coherent and noncoherent space-time receivers," IEEE Trans. Inf. Theory, vol. 47, no. 6, pp. 2383-2399, Sept. 2001.

[27] X.-B. Liang and X.-G. Xia, "Unitary signal constellations for differential space-time modulation with two transmit antennas: parameteric codes, optimal designs, and bounds," IEEE Trans. Inf. Theory, vol. 48, no. 8, pp. 2291-2322, Aug. 2003.

[28] Z. Liu, G. B. Giannakis, and B. L. Hughes, "Double differential space-time block coding for time-varying fading channels," IEEE Trans. Commun., vol. 49, pp. 1529-1539, Sept. 2001.

[29] A. Shokrollahi, B. Hassibi, B. M. Hochwald, and W. Sweldens, "Representation theory for high-rate multiple-antenna code design," IEEE Trans. Inf. Theory, vol. 47, pp. 2335-2367, Sept. 2001.

[30] H. Li and J. Li, "Differential and coherent decorrelating multiuser receivers for space-time-coded CDMA systems," IEEE Trans. Signal Process., vol. 50, no. 10, pp. 2529-2537, Oct. 2002.

[31] E. Zimmermann, P. Herhold, and G. Fettweis, "On the performance of cooperative diversity protocols in practical wireless systems," in Proc. IEEE Veh. Technol. Conf. 2003, pp. 2212-2216.

[32] Q. Zhao and H. Li, "Performance of differential modulation with wireless relays in Rayleigh fading channels," IEEE Commun. Lett., vol. 9, no. 4, pp. 343-345, Apr. 2005. 
[33] P. Tarasak, H. Minn, V. K. Bhargava, "Differential modulation for two-user cooperative diversity systems," in Proc. Globecom 2004.

[34] D. Chen and J. N. Laneman, "Modulation and demodulation for cooperative diversity in wireless systems," submitted to IEEE Trans. Wireless Commun.

[35] X. Li, "Space-time coded multi-transmission among distributed transmitters without perfect synchronization," IEEE Signal Process. Lett., vol. 11, no. 12, pp. 948-951, Dec. 2004.

[36] Y. Hua, A. Swami, and B. Daneshrad, "Combating synchronization errors in cooperative relays," in Proc. IEEE ICASSP 2005.

[37] Y. Zhang, G. Wang, and M. G. Amin, "Imperfectly synchronized cooperative network using distributed space-frequency coding," in Proc. IEEE Veh. Technol. Conf. 2005.

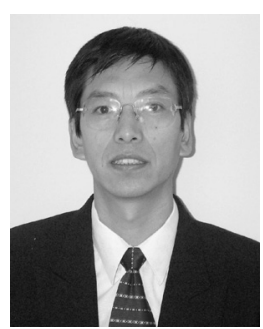

Genyuan Wang received the B.Sc. and M.S. degrees in mathematics from Shaanxi Normal University, Xi'an, China, in 1985 and 1988, respectively. He received his Ph.D. degree in electrical engineering from Xidian University, Xi'an, China, in 1998.

From 1988 to 1994, he worked at Shaanxi Normal University as an assistant professor and then an associate professor. From 1994 to 1998 , he worked at Xidian University as a research assistant. He worked with the Department of Electrical and Computer Engineering, University of Delaware as a post-doctoral fellow. Currently, he works with the Center of Advanced Communications, Villanova University, as a research associate. His research interests are radar imaging, radar signal processing, adaptive filter, OFDM system, channel equalization, channel coding, space-time coding, and multiple-input multiple-output for broadband wireless communication systems.

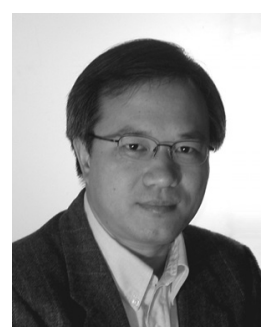

Yimin Zhang (SM'01) received his Ph.D. degrees from the University of Tsukuba, Tsukuba, Japan, in 1988. He joined the faculty of the Department of Radio Engineering, Southeast University, Nanjing, China, in 1988. He served as a Technical Manager at the Communication Laboratory Japan, Kawasaki, Japan, from 1995 to 1997, and was a Visiting Researcher at ATR Adaptive Communications Research Laboratories, Kyoto, Japan, from 1997 to 1998. Since 1998, he has been with the Villanova University, where he is currently a Research Associate Professor at the Center for Advanced Communications and the Director of the Radio Frequency Identification (RFID) Lab.

Dr. Zhang's research interests are in the areas of array signal processing, space-time adaptive processing, multiuser detection, MIMO systems, ad hoc and cooperative communications, blind signal processing, digital mobile communications, and time-frequency analysis. He is an associate editor for the IEEE Signal Processing Letters.

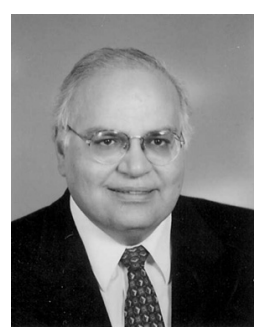

Moeness G. Amin (F'00) received his Ph.D. degree in 1984 from University of Colorado, Boulder. He has been on the Faculty of Villanova University since 1985, where is now a Professor in the Department of Electrical and Computer Engineering and the Director of the Center for Advanced Communications.

Dr. Amin is the recipient of the IEEE Third Millennium Medal; Distinguished Lecturer of the IEEE Signal Processing Society for 2003-2004, Member of the Franklin Institute Committee of Science and Arts; Recipient of the 1997 Villanova University Outstanding Faculty Research Award; Recipient of the 1997 IEEE Philadelphia Section Service Award. Dr. Amin has over 280 publications in the areas of Wireless Communications, Time-Frequency Analysis, Smart Antennas, Interference Cancellation in Broadband Communication Platforms, Digitized Battlefield, Direction Finding, Over the Horizon Radar, Radar Imaging, and Channel Equalizations. 